\title{
SELECCION DE GERMOPLASMA DE FREJOL COMÚN (Phaseolus vulgaris) Y CHICHARRO (Vigna unguiculata) RESISTENTES A Rhizoctonia solani
}

\section{(Selection of common beans(Phaseolus vulgaris) and cowpea (Vigna unguiculata) germoplasms resistant to Rhizoctonia solani)}

\author{
M.A. Noronha; S.J. Michereff \& R.L.R. Mariano \\ Area de Fitossanidade. Departamento de Agronomia Universidade Federal \\ Rural de Pernambuco 52 171-9)()(). Recifc. PE. Brasil, E-mail: sami(a): truenct.com.br
}

Palabras clave: resistencia varictal. Rhizoctonia soluni. frejol común. chicharro

Key words: varictal resistance. Rhizoctonia soluni. common beans. cowpea

\section{RESUMEN}

Con la finalidad de seleccionar fuentes de resistencia a Rhizoctor ia solani entere germoplasmas de fiejol cominy chicharro, se efectuaron evaluaciones en condiciones de invernadere y campo. En la primera situacionn, 20) germoplasmas de cada leguminosa fueron evaluados. Las semillas fueron sembradas en stedo esterilizado, previamente infestado can el parógeno. mediante la incorperación de $50 \mathrm{mg}$ de sustrate colonizade (anroz) $\mathrm{kg}$ de suclo.

La evaluación de les germoplasmas fire realizada 1.5 dias despuéscle la siembra, mediante una escalade valores muméricos, siendo distrihuidas en las categoriasde" "resistente, moderadamente susceptible (mod. susce) y susceptible". Tres germoplasmas de fiejol (1.11.53-53-, IP.1-5e IP.1-9), se comportaron comor resistentesa $R$. solumi, mientras que entre los de chicharro, seobservaron reacciones mod. susc: y susceptibles: lin las condiciones de campo, en las huertas que comtenian suelo previamente infestado por el patogeno (10g $\mathrm{m}^{2}$ de suelor), se evaluaron 7 germoplasmas de fiejol y 13 de chicharro.

La severidad de la enfermedad fire evaluada 21 dias después de lasiembra, conforme a lamérodologia unilizada en invernadero. Todes los germoplasmas de fiejol y 3 germoplasmas de chicharro (comujinha. LIO)1.0)(0)-2 y. L191.006) se commportan'on como mod. susce, mientras que los demás fireron clasificules como susceptibles.

Los resultados evidenciaron la dificultad en la obtencioin de germoplasmas con altos niveles de resistencia a $R$. solani, indicande lanecesided de laimplementercirin de prácticas integradas que conduzcan al contitos de la enferemedad, en la cual el usis de germoplasmas conn resistencia mod. susce seria uno de lose compenentes.

\section{SUMMARY}

With the purpose of selecting sontces resistant to Rhizoctoria soluni anong germoplasms of beans and conpea, evaluations in greenhouse and field conditions werecarried out. Inthefirst case, that isunder green-house conditions, 20 germoplasms fiom each legume were evaluated.

Seedswere planted insterilized soil previously infested with the pathogen through the addition of $50 \mathrm{~m}$. colonized substrate (rice) Kg of soil. The evaluation of germoplasms wasperformed 15 dansafierplantation by the use of a grade scale wich classified inem as "resistant, moderately susceptible (mod.susc.) andsusceptible".

Three germoplasms of hean (AN53-53 t, IPA-5 and $I P A-9)$ showed themselves resistant to $R$. solani, whereas moderately susceptible and susceptible reactions were present anomg those of conpea. Underfield conditions, 7 germoplasms of beans and 13 of compea were evaluated in orchards having a soil previously infested with the pathogen ( $10 \mathrm{~g} \mathrm{~m}^{2}$ of soil).

Disease severity was assessed 21 days afier the planting, according to the method employed under greenhouse conditions. All germo-plasmis of bean and three germoplasms of conpea (Corujimha, L101.000-2 and L.191.006) behavedasmode-ratelysusceptible while the rest was classified as susceptible.

Results showed hat is ver difficult to get germoplasms highty resistant to R. solumi, so there is aneed to implement integrated practices, which include the use of imoderately sisceptible resisting germoplasms asone of the components andleatro the disease control. 


\section{INTRODUCCION}

Las producciones de frejol común (Phaseolus vulgaris L.) y de chicharro (Vigna unguiculata (L.) Walp.). son afccladas por muchos factores cdílicos. climáticos y bióticos. Sin cmbargo. en la may oría de las zonas productoras. la alta presión de enfermedades constituye uno de los factores que reducen mís significaltivamente los rendimientos a nivel de predio (Becbe \& Pastor-Corrales. 1991: Schoonhoren \& Vorsest. 1994: Pio-Ribeiro \& Assis Fillıo. 1997).

La pudrición radical. causada por Rhizoctonia solani Kühn, es una de las enfermedades más comunes del frejol y del chicharro cu cl Nordeste del Brasil y en el mundo (Rios. 1990); Abawi. 1994: Schoonhoren \& Voy sest. 1994). ocasionando pudriciones de semillas. Iesiones cóncalvas rojizopardas y cancros en el hipocótilo. culminando con la caidal y muerte de las plántulas (Cardoso. 1994). El hongo se encuentra distribuido en la may oría de los suclos agricolas. condiversos niveles de infestación. y puede afeclar muchas especies de plantas (Baker. 197()). Aunque la información sobre los efectos de la enfermedad en el rendimiento de los cultivos en regiones productorias es limitadia. se sabe que las pérdidas pueden ser considerables y que a menudo varian entre los suclos de unia misma zona. como también dentro del mismo predio de una siembra a otra (Abawi \& Pastor-Corrales. 1990). Infecciones cercantas al $10(0 \% \%$ y pérdidas casi totales cn cultivos de frejol. fucron obscriadas en Colombia. Perú y USA (Pastor-Corrales \& Abawi. 1988). En Brasil. pérdidas cercanas al $6(0 \%$ se han registrado en frẹjol juntocon la pudrición radical causada por Fusarium soluni .sp. phaseoli (Hagedorn. 1991). En chicharro. Cardoso el al. (1991) relataron pérdidas de $6(1) \% \mathrm{cnl}$ la producción del cultivar BR-I Poly en Brasil. micntrasque en Nigeria, junto con Pythium aphanidermatum cl $75 \%$ de la mortalidad de las plántulas de chicharro se produjo durante las tres semanas después de la siembra (Williams. 1975).

La v rabilidad del mancjo de las pudriciones radicales del frejol y chicharro. se ha demostrado mediante implementación de estrategias de control integrado. tales como uso de cultivares resistentes. rolición de cultivos y tratamicnto de semillas o suclos con fungicidas. (Rios. 1988: Abawi \& Pastor-Corrales. 1990): Abawi. 19yt: Cardoso. 1994: Zambolim ("r al.. 1997). Sin cmbargo. cl uso de cultivares resistentes es la estrategia más efectiva para el control de enfermedades. especialmente para los agricultores de países en desarrollo y con pocal disponibilidad de insumos o capital (Abawi. 19y4: Rios. 1988).

Han sido identificados germoplasmas de frejol con un buen grado de resistencia (Abawi \& Pastor-Corrales. 199): Becbe \& Pastor-Corrales. 1991: Abawi. 1994). sin embargo. cultivares comerciales de frejol y chicharro adalplados y resistentes a $R$. solani no estin disponibles (Hagedorn.
199) 1: Cardoso. 1994: Pinnella \& Ruppel. 1996: Pio-Ribciro \& Assis Fillo. 1997).

El objectivo de csta inr estigación. fue evaluar en condiciones de invernadero y campo, la resistencia frente a $R$. solani. de germoplasmas de frejol y chicharro.

\section{MATERIALES Y METODOS}

Fitopatógeno e inóculo. Sc utilizó una ccpade $R$. solani (RS-3). proveniente de planta de frejol común con sintomas de cancro en el hipocótilo y procedente de un área de cultivo comercial del Estado de Pernambuco. La cepa pertenece al grupo de anastomosis + ( $\mathrm{AG}-4$ ) determinado conforme a la metodología descrita por Bolkan \& Ribciro(1985). El inóculo del fitopatógeno fue preparado en sustrato constituido de arro\% sin cáscara autoclavado según los procedimientos descrilos por Barbosa é al. (1995). Alicuotas del inóculo fucron pesadas conforme a la concentración a utilizarse en cl suclo.

Selección preliminar de germoplasmas resistentes a R.soluni. En condiciones de invernadero se evaluaron 20) germoplasmas de frejol y 20 de chicharro. Las semillas fueron desinfectadas con solución de $\mathrm{NaClO}(1.5 \%)$, lavadas con agua destilada cstćril y luego secadas. La siembra se éfectuó cn bandejas plásticas ( $3($ ) $\times 2.3 x+$ cm y capacidad de $2 \mathrm{~kg})$. las cuales contenían suclo esterilizado $(\mathrm{pH} 6,8$; matcrial orgínica $3.5: \mathrm{N} 1.285 \mathrm{ppm}: \mathrm{P}>36 \mathrm{ppm} ; \mathrm{K}>250 \mathrm{ppm}$ : $\mathrm{Al}\left(0.03 \mathrm{mcq} / 10\left(0 \mathrm{~cm}^{3} ;\right.\right.$ Ca $7.6 \mathrm{mcq} / 100() \mathrm{cm}^{3}: \mathrm{Mg} 1.12 \mathrm{mcq} / 100$ $\mathrm{cm}^{3}$ ). previamente infestiddo con el sustrato colonizado por R. solami. cn concentración de 5() $\mathrm{mg} / \mathrm{kg}$ de suclo. El discrio cxperimental fue completamente al azar. con 10 repeticiones. donde cada repetición consistia de una bandeja con 25 semillias.

La evaluación de la intensidad de la enfermedad se realìó a los 15 dias después de la sicmbri. utilizándose una escalia de valores que fluctúo entre 1 y9) (Schoonhoren \& Pastor-Corrales. 1987), donde:

$1=\sin$ sintomas visibles.

3 = decolonación ligeral. ya sea sin lesiones necróticas ocon un 10\%:aproximadamente de los tẹidos del hipocótilo Y de la rail cubicrtas con lesiones.

$5=$ aproximadamente $2.5 \%$ de los tejidos del hipocótilo y de la rair cubiertas con lesiones. pero los tejidos se conservan firmes y hay poco deterioro del sistema radical.

7 = aproximadamente $50 \%$ de los tejidos del hipocótilo y de la rair cubiertas con lesiones que se combinan con ablandamiento. pudrición y reducción considerables del sistema radical.

$9=$ aproximadamente $75 \%$ o más de los tejidos del hipocótilo y de la raiz afectados por estados avanzados de pudrición. en combinación con una reducción severa del sistemia radical. 
Los germoplasmas fueron clasificados conforme a los valores obtenidos. donde de $1 .($ ) a 3.5 se considerann 'resistentes', de 3,6 a $6,5^{\circ}$ moderadamente susceptibles (mod. susc.)' y de 6.6 a $9 .($ ) 'susceptibles".

Durante el periodo de ejecución de los experimentos. la temperatura en el invernadero fuede $39 \pm 3,1$ " $\mathrm{C}$ y la humedad relativa de $67 \pm 2.7 \%$. obtenida mediante un termohigrógrafo.

Selección de germoplasmas resistentes a $R$. solani en condiciones de campo. Se craluaron scis germoplasmas de frejol (IPA-1. IPA-5. IPA-8. IPA-9). NA-53+-53+. Gordo) y seis de chicharro (IPA-205. L.+3.0(0)-1. L.9+.(0)(0)-3.

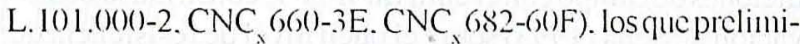
narmente presentaron un mayor potencial como fuentes de resistencia a $R$. solani. Adicionalmentc. scis germoplasmas nativos de chicharro (Corujinhia. Sempre Verde. Rasga Letra. Branco. Travessiay Canapu) fucron inclúidos en los ensayos. Los germoplasmas L. I(0). 117 y L. 19) 1.0()6. respectivamente de frcjol y chicharro. fucron utilizados como patrones de susceptibilidad. basado en la selección preliminar. Las semillas fucron desinfectadas y sembradas en hucrtas ( $1.2 \mathrm{~m}$ de largo $10 .(0) \mathrm{m} \times() .8 \mathrm{~m})$. lascuales contenian suclo no cstcrilizado ( $\mathrm{pH} 6,5$ : materia orgínical $2.3: \mathrm{N} \mathrm{IO} 10)$ ppm: $\mathrm{P}>36 \mathrm{ppm}: \mathrm{K}>2.5(1) \mathrm{ppm}: \mathrm{Al}(0.00) \mathrm{meq} / 10\left(0 \mathrm{~cm}^{3}: \mathrm{Ca} 6.3\right.$ $\mathrm{mcq} / 1(0) \mathrm{cm}^{3}: \mathrm{Mg}\left(0.97 \mathrm{mcq} / 1(0) \mathrm{cm}^{3}\right)$. previamente infestado con cl sustrato colonizaldo por $R$. solani. en concentración de $10 \mathrm{~g} / \mathrm{m}^{2}$ de suclo.

El discño cxperimental fue de bloques completamente al azar. con cinco repeticiones. donde cadda repet ición consistia de $1 \mathrm{~m}^{2}$ de suclo con 16 surcos de siembra.

La craluación de la intensidad de la enfermedad se realizó a los 15 dias después de la siembra. conforme a lo descrito anteriormente.

Durante el período de cjecución del experimento. la temperatura ambiente fue de $3.3 \pm 2.2^{\circ} \mathrm{C}$ y la humedad relativa de $69 \pm 3.2 \%$. obtenida mediante un termohigrógrafo inslalado a $300 \mathrm{~m}$ de las parcelas.

\section{RESULTADOS Y DISCUSION}

En la selección preliminar. tres germoplasmas de frcjol (AN 534-6.34. IPA-5 y IPA-9) se comportaron como resistentes a R.solani. mientras que 15 fueron moderadamente sensibles y dos susceptibles (Tabla 1). Los resultados dificren de Suguino \& Maringoni ( 1995 ). que al cialuar cl comportamento de germoplasmas de frejol en relación a R. soluni en condiciones de invernadero. verilicaron que todos se comportaron como susceptibles. presentando elerado índice de sereridad de la enfermedad. inclusive IAPAR-It y Carioca que evidenciaron una reacción moderadamente susceptible en el presente estudio en inrernat- dero. Con relación a chicharro, ningún germoplasma evidenció resistencia a $R$. solani. aún cuando 17 se comportaron como mod. susc. y tres como susceptibles (Tabla 2).

Los resultados evidenciaron que $R$. solani perjudicó notablemente el desarrrollo de las plantas, principalmente en cl estado inicial de crecimento, confirmando las observacioncs de Rios (1988) y Cardoso (1994), de que frejol y chicharro son susceptibles a este patógcno a partir de la germinación. siendo csta susceptibilidad inversamente proporcional al desarrollo de la planta.

Tabla 1. Reacción de germoplasmas de frejol común a R. solani en condiciones de invernadero.

\begin{tabular}{|c|c|c|c|}
\hline $\begin{array}{l}\text { Germo- } \\
\text { plasmat }\end{array}$ & $\begin{array}{l}\text { Proce- } \\
\text { dencia }\end{array}$ & $\begin{array}{l}\text { Gradode } \\
\text { Infección }\end{array}$ & Reacción $^{3}$ \\
\hline AN534-534 & CNPAF & 3,1 & Resistente \\
\hline IPA-5 & IPA & 3,3 & Resistente \\
\hline IPA-9 & IPA & 3,4 & Resistente \\
\hline IPA-1 & IPA & 3,7 & Mod. susc. \\
\hline IPA-8 & IPA & 3,9 & Mod. susc. \\
\hline Gordo & IPA & 3,9 & Mod. susc \\
\hline Boi Dcitado & IPA & 4,5 & Mod. susc. \\
\hline IPA-7 & IPA & 4,7 & Mod. susc. \\
\hline L. 162.012 & IPA & 4,8 & Mod. susc. \\
\hline IPA-11 & IPA & 5,0 & Mod. susc. \\
\hline IPA-10 & IPA & 5,5 & Mod. susc. \\
\hline Mão Curta & IPA & 5,8 & Mod. susc. \\
\hline IPA-3 & IPA & 5,9 & Mod. susc. \\
\hline L.94.133 & IPA & 6,1 & Mod. susc. \\
\hline IAPAR-I4 & IAPAR & 6,1 & Mod. susc. \\
\hline AN 512-722 & CNPAF & 6,1 & Mod. susc. \\
\hline Carioca & IPA & 6,3 & Mod. susc. \\
\hline TY.3364.15 & CIAT & 6,5 & Mod. susc. \\
\hline L. 100.117 & IPA & $6,6 \mathrm{~m}, \mathrm{l}^{\circ}$ & Susceptible \\
\hline Favita & IPA & 6,8 & Susceptible \\
\hline
\end{tabular}

${ }^{\prime}$ C.V'? IF Centro Nacional de Investigaciones de Aroz y Frejol - EAMBR.1P.1, Goicinia, G(), Brasil; IP.1 = Empresa Penambucanade Invessigación Igropecuaria, Recife, PE. Brasil; I.IP'.IR = Instituro.Igronómicodel Paraná, Londrina, PR, Brasil; C1.1T Centrolmennacionalde. Igriculnura Tropical, Cali, Colombia. "Imensidad de la enfermedad onforme mana escala de valores que varia de la9) (Schoromhoren of P'astor-Corrales, 1987). 1 Mediade l() repeticiones. "Gradosderesistencia: $1,0-3,5=$ resistente; $3,6-6,5=$ mod. susc.; $6,6-9,0=$ susceptible.

En condiciones normales. esta resistencia natural se alcanzal entre 20) al 25 dias después de la siembra (Cardoso, 1994). Las plantas más viejas se tornan resistentes a la infección de $R$. solani posiblemente debido a un incremento en el contenido de calcio en sus tẹidos, a la inducción de 
fitoalcxinas, y/o a la disminución cn los cxudados del hipocótiloy de la raiz. los cuales cstimulan la formación del cojín de infección por parte del hongo (Dodman \& Flentje. 1970; Stockwell \& Hanchey, 1984). Además. Broglic et al., (1991) y Benhamou et al. (1993). utilizando plantas transgénicas, verificaron que niveles crecientes de endoquitinas aum entaban la resistencia a $R$. solumi.

Tabla 2. Reacción de germoplasmas de chicharro a $R$. solani en condiciones de invernadero

\begin{tabular}{|llll|}
\hline $\begin{array}{l}\text { Germo- } \\
\text { plasma }\end{array}$ & $\begin{array}{l}\text { Proce- } \\
\text { dencia }^{1}\end{array}$ & $\begin{array}{l}\text { Grado } \\
\text { Infecc. }^{2}\end{array}$ & Reacción $^{3}$ \\
\hline IPA-205 & IPA & 4,9 & Mod. susc. \\
L.43.000-1 & IPA & 5,1 & Mod. susc. \\
CNC $682.60 F$ & CNPAF & 5,2 & Mod. susc. \\
L.101.000-2 & IPA & 5,4 & Mod. susc. \\
L.94.000-3 & IPA & 5,4 & Mod. susc. \\
CNC $660-3 E$ & CNPAF & 5,5 & Mod. susc. \\
L.97.000-1 & IPA & 5,7 & Mod. susc. \\
L.198.005 & IPA & 5,7 & Mod. susc. \\
L.198.003 & IPA & 5,9 & Mod. susc. \\
L.145.001 & IPA & 6,0 & Mod. susc. \\
L.145.002 & IPA & 6,1 & Mod. susc. \\
CNC $18135 C_{2}$ & CNPAF & 6,1 & Mod. susc. \\
IPA-206 & IPA & 6,2 & Mod. susc. \\
L. $100.000-4$ & IPA & 6,2 & Mod. susc. \\
CNC $679-44 E$ & CNPAF & 6,3 & Mod. susc. \\
L.174.011 & IPA & 6,3 & Mod. susc. \\
L.94.002 & IPA & 6,6 & Mod. susc. \\
L.142.001 & IPA & 6,9 & Susceptible \\
L.190.010 & IPA & 7,1 & Susceptible \\
L.191.006 & IPA & 7,2 & Susceptible \\
\hline
\end{tabular}

'IP.A = Empresa Pernambucana de Investigación. I gropecuaria, Recife, PE, Brasil; CNP'AF = Centro Nacional de Investigación de Arroz y Frijol-EA IBR.AP.A, Goicinia, GO, Brasil. Intensidad de la enfermedad conforme ana escala de valores que variande? a9 (Schoonhoven \& Pastor-Corrales, 1987). Mediade 10 reperticiones. ${ }^{3}$ Gradosderesistencia: $1,0-3,5=r$ esistente; $3,6-6,5=$ mod. susc.; 6, 6-9,0= susceptible.

La coloración oscura del tegumento de las semillas ha sido relacionada a la resistencia del frcjol a $R$. solani (Dcakin \& Dukes. 1975: Prasad \& Weigle. 1976: Guevara-Torres et al., 1986: Camara et al. 1988). No obstante, en el presente estudio, con excepción del cultivar IPA-10) que posec tegumento negro, las demás presentaban tegumento calé o marrón. sin quedar er idenciadal la importancia de la coloración del tegumento. considerando que varios germoplasmis presentaron desempeño superior a IPA-10. La ausencia de una estrecha relación entre materiales de semilla negray la resistencia a $R$. solani también ha sido registrada en otras investigaciones (Becbe et al., 1981; Abawi, 1994; Suguino \& Maringoni, 1995). Al evaluarse bajo condiciones de campo, todos los germoplasmas de frejol presentaron nivel moderadamente susceptibles de resistencia a $R$. solani (Tabla 3). siendo diferente a lo verificado en la selección preliminaren relación a los germoplasmas AN 534-534, IPA5 y IPA-9. cuando se comportaron como resistentes, así como en relación al germoplasma L.100.117, que había evidenciado susceptibilidad. El resultado obtenido en condiciones de campo con el cultivar IPA-1, confirma la observación de Sena (1993) sobre cl buen nivel de resistencia de cste germoplasma en relación a $R$. solani.

Tabla 3. Reacción de germoplasmas de frejol común a $R$. solani en condiciones de campo.

\begin{tabular}{|lcl|}
\hline Germoplasma & $\begin{array}{c}\text { Grado de } \\
\text { Infección }\end{array}$ & Reacción ${ }^{2}$ \\
\hline IPA-1 & 3,7 & Mod. susc. \\
Gordo & 3,9 & Mod. susc. \\
L. 100.117 & 3,9 & Mod. susc. \\
IPA-8 & 4,3 & Mod. susc. \\
AN 534-534 & 4,3 & Mod. susc. \\
IPA-5 & 4,4 & Mod. susc. \\
IPA-9 & 5,2 & Mod. susc. \\
\hline
\end{tabular}

Imensidad de la enfermedad conforme una escala de valores que varia de 1 a 9 (Schoonhoven \& Pastor-Corrales, 1987). Media de 5 repeticiones. "Grados de resistencia: 1,0-3,5= resistemle; $3,6-6,5=\bmod$. susc,$; 6,6-9,0=$ susceptible.

Entre los germoplasmas de chicharro. apenas tres fueron clasificados como mod. susc. en condiciones de campo (Corujinha, L101.00(0)-2 y L191.0(6)), mientras que los demás como susceptibles a $R$. solani (Tabla 4). Como fue verificado en frejol, en condiciones de campo, varios germoplasmas de chicharro presentaron reacciones diferentes a las observadas en invernadero. incluyendo el pat rón de susceptibilidad (L. 191.0(6)). La mayor susceptibilidad de germoplasmas de chicharro a $R$. solani, tambićn fue constatada por Sumner (1985). ya que ninguno de los principales cultivares de esta leguminosa plantados en Gcorgia ( USA), fuc resistente al patógeno.

Algunas investigaciones han evidenciado la baja correlación entre resultados obtenidos en invernadero y campo (Camaratet al. . 1988; Pastor-Corrales \& Abawi, 1988), cn forma similar a lo encontrado en el presente estudio. 
Varios factores pueden contribuir para cstas diferencias en la reacción de germoplasmas, incluyendo cambios en la fisiología de la planta, velocidad de emergencia y vigor de la plántula, características del inóculo y condiciones ambientales (Pastor-Corrales \& Abawi, 1988: Weinhold \& Sinclair, 1996).

Tabla 4. Reacción de germoplasmas de chicharro a $R$. solani en condiciones de campo.

\begin{tabular}{|c|c|c|}
\hline Germoplasma ${ }^{1}$ & $\begin{array}{l}\text { Gradode } \\
\text { Infección }^{2}\end{array}$ & Reacción ${ }^{3}$ \\
\hline L.191.006 & 5.9 & Mod. susc. \\
\hline Corujinha* & 5,9 & Mod. susc. \\
\hline L. $101.000-2$ & 6.2 & Mod. susc. \\
\hline L.94.000 & 6.6 & Susceptible \\
\hline IPA-205 & 7.1 & Susceptible \\
\hline $\mathrm{CNC}_{x} 682-60 \mathrm{~F}$ & 7,2 & Susccptible \\
\hline Canapu* & 7,3 & Susceptible \\
\hline Travessia* & 7,3 & Susceptible \\
\hline $\mathrm{CNC}_{x} 660-3 \mathrm{E}$ & 7.6 & Susceptible \\
\hline L. $43.000-1$ & 7,9 & Susceptible \\
\hline Rasga Letra* & 7,9) & Susceptible \\
\hline Sempre Verde* & 8.0 & Susceptible \\
\hline Branco* & 8,1 & Susceptible \\
\hline
\end{tabular}

${ }^{\prime}$ Germoplasmas seguidos de asterisco $\left({ }^{*}\right)$ son procedentes del Centro de Asesoria y Apovo a los Trabajadores e Instituciones No Gubernamentales Alternativas - C.H.1TING.1, Ouricuri, PE, Brasil. Intensidad de la enfermedad conforme una escala de valores que varia de 1 a 9 (Schoonhoven \& Pastor-Corrales, 1987). Media de 5 repeticiones. "Grados deresistencia: 1,0-3,5= resistente; $3,6-6,5=$ mod. susc:; $6,6-9,0=$ susceptible.
La dificultad en la obtención de fuentes conaltos niveles de resistencia a $\boldsymbol{R}$. solani en chicharro y frejol, como lo observado en el presente cstudio, también fue relatado por otros investigadores (Abawi \& Pastor-Corrales, 1990; Abawi, 1994; Cardoso, 1994; Suguino \& Maringoni, 1995). Solamente niveles bajos a moderados de resistencia han sido detcctados para fitopatógenos del suelo no especializados, entre los cuales figura $R$. solani, como consecuencia de la pequeña diferencia en la susceptibilidad de los germoplasmas, de la existencia de amplia gama de hospederos y de la alta competitividad y rápida colonización de la materia orgánica del suclo (Abawi \& Pastor-Corrales, 1990; Akino \& Ogoshi, 1995).

Algunos germoplasmas de frejol que evidenciaron resistencia moderada a $R$. solani en el presente estudio, se utilizaron como parentales en cruzamientos, debido a otras características deseables, como por ejemplo, IPA-5 es resistente a la roya (Uromyces appendiculatus), Gordo es resistente a la roya y antracnosis (Colletotrichum lindemuthiamum), mientras que IPA-8 es resistente a la roya, antracnosis y la marchitez debida a Fusarium (Fusarium oxysporum f.sp. phaseoli) (Sena, 1993). Debido a la ausencia de cultivares con altos niveles de resistencia a $R$. solani, los germoplasmas de frejol y chicharro con moderados niveles de resistencia detectados en este estudio, podrían ser utilizados en un programa de manejo integrado de la enfermedad, en el que los niveles del inóculo del patógeno serían reducidos antes de la plantación por la implementación de prácticas de cultivo, como siembras a poca profundidad, rotación con plantas no hospedantes como maíz cnmienda del suelo con un material que se pueda descomponer o mediante la incorporación de residuos selcccionados y arado profundo del suelo.

\section{REFERENCIAS}

Abawi, G.S. (1994). Pudriciones radicales. In: Pastor-Corrales. M. \& Schwart\%. H.F. eds. Problemas de produceion del liijol en los trópicos. 2. ed. Centro Internacional de Agricultura Tropical. Cali. pp. $121-184$

Abawi, G. S. \& Pastor-Cormales, M. A. (199(0). Root rots of beans in Iatin America and Africa: diagnosis, resuarch methodologies, and manengement stralegies. Centro Intemacional de Agricultura Tropical, Cali. pp.83-99

Akino, S. \& Ogoshi, A. (1995). Pathogenicity and host specilicity in Rhizoctonia solani. In: Kohmoto, K.: Singh. U.S. \& Singh. R.P. eds. Pathogenesis and host specilicity in plant discases Histopathological, biochemical, genetic and molecular bases. II: Eukaryotes. Pergamon/Elsevier Science. Kidlingloti. pp.37-49

Baker, K.F. (1970). Types of Rhizocronia discases and their occurrence. In: Pammeter. J.R. ed. Rhizoctonia solani: biology and pathology. The University of California Press, Berkeley. pp.125148

Barbosa, M.A.G.; Michereff, S.J.; Mariano, R.L.R. \& Maranhão, E. (1995). Biocontrole de Rhizoctonia solani em caupi pelo tratamento de sementes com Pseudomonas spp. lluorescentes. Summa Phytopathologica 21:151-157

Becbe, S.E. \& Pastor-Corrales, M. (1991). Breeding for disease resistance. In: Van Schoonhoven, A. \& Voysest, O. eds. Common beans: Research for crop improvement. CAB International, W'allingford. pp.561-617

Becbe, S.E.; Bliss, F.A. \& Schwartz, II.F. (1981). Root rot resistance in common bean germ plasm of Latin American origin. Plant Dis. 65:485-489

Benhamou, N.; Broglie, K.; C.het, I. (1993). Antifungal effect of 
bean endochitinase on Rhizoctonia solani: ultrastructurial changes ane cytochemical aspects of chitin breakdown. Canadian Journal of Microbiology 39:318-328

Broglie, K.; Chet, I.; Ilolliday; N.; Cressman, R.; Biddle, P.; Knowlton s.; Mauvais, C..J. \& Broglie, R. (1991). Transgenic plants with enhanced resistance to the fungal pathogen Rhizoctonia solani. Science 254:1194-1197

Camara, M.; IIerrera-Isla, L.; Galantai, L. \& Guevara-Torres, V. (1988). Resistencia varietal a Rhizoctonia solani Kühn en firijol y soya. Revista Centro Agricola 15:24-29

Cardoso, J.E.; Pozer, I.; Bevitori, R. \& Virgens, D... (1991). Avaliação de perdas no feijào-de-corda ( İ̈gna unguiculata (L.) Walp.) causadas pela podridão radteular de Rhizoctonnia (R. solani Kühn). In: Reunião Nacional de Pesquisal de Caupi, 3.. Fortalěa. ClE, 1991. Imprensa Universitiria - UlC. liortaleza. pp.51

Cardoso, J.E. (1994). Podridōes radiculares. In: Sartoralto, A. \& Rava, C.A. eds. Principais doençäs do leijociro comum s seu controle. EMIBRAPASPI, Brasilia. pp.151-164

Deakin, J.R. \& Dukes, P.D. (1975). Breceling snap beans for resistance to discalse cilused by Rhisoctonnia solani Külnn. HortScience 10:269-271

Dodman, R.L. \& Flentje, N.T. (1970). The mechanism and physiology of plant penctration by Rhizoctomia solemi. In: Parmeter, J.R. ed. Rhizoctonia solami: biology and pathology. The liniversity of California Press. Berkeley. pp. 1+0)-160)

Guevara-Torres, V.; IIerrera-Isla, L.; Cammara, M. \& Galantai, E. (1986). El color de la semilla del frijol (Phaseolus volgaris L.) y su relación con la resistencia al at:aque de Rhizoctonia solani Kühn. Revista Centro Agricola 13:3-6

Hagedorn, D.J. (1991). Rhizoctonia root rot. In: Hall, R. ed. Compendium of bean diseases. St. P'aul: APS' Press, 1991. p.13

Panclla, \. \& Ruppel, E.(. (1996). Availability of germplasm for resistance against Rhizoctonia spp. In: Sneh. B.: Jabaji-IIare. S.: Neate. S. \& Dijst, G. eds. Rhizoctomia species: taxonomy, moleular biology. ecology: pathology and diseatse control. Klawer Academic Publishers, Dordrecht. pp.515-527

Pastor-Corrales, Mr.A. \& Abawi, (i.S. (1988). Bean atcessions with resistance to Rhizoctonia solani under lield conditions in Colombia. Turrialba 38:83-86

Pio-Ribeiro, G. \& Assis Filho, F.M. (1997). Doençăı do caupi (V̈̈gna unguiculata (L.) Walp.). In: Kimati, II.; Amorim, L.; Bergamin lïlho, A.: Camargo. L...... \& Re\%ende. J.A.M. eds. Manual

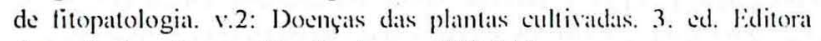
Agronòmica Ceres. São Pautolo. pp.23.3-2.4
Prasad, K. \& Weigle, J.I. (1976). Association of seed coat factors with resistance to Rhizoctonia solani in Phaseolus vulgaris. Phytopatthology $66: 342-345$

Rios, (. P. (1988). Doençăs lüngicas e bacterianas de caupi. In: Araújo. J.P.I' \& W'alt. l:.l: eds. O caupi no Brasil. IITAEMBRAPA, Brasilia. pp.547-589

Rios, (i.P. (1990). Principais doençass do caupi no Brasil. EMIBRAP'A-C'NPAP, Goiania.

Schoonhoven, A. Van \& Pastor-Corrales, M. (1987). Sistema estándar para la evaluacion de germoplasma de frijol. Centro Internacional de Agricultura Tropical, Cali.

Schoonhoven, A. Vian \& Voysest, O. (1994). El frijol comin en America Latina y sus limitaciones. In: Palstor-Corrales. M. \& Schwart\%. H.F. eds. Problemas de producioin del frijol en los trópicos. 2. ed. Centro Internacional de Agricultura Tropical, Cali. pp.39-66

Sena, R.C..(1993). Caracteristicas ticnicals de culturas de importaincia para al conomia de Pernambuco. Il'A. Recife.

Stockwell, V.O. \& IIanchey, P. (1984). The role of the cuticule in resistance of beans to Rhizoctonia solani. Phytopathology 74:1640-1642

Suguino, L.\&. Maringoni, A.C. (1995). Comportamento de variedades de leijociro a Rhizoctomia solani. Summat Phytopathologica 21:124-126

Sumner, D. (1985). Virulence of antastomosis groups of Rhizoctonia solani and Rhizoctomiatlike fungi on selected germplasm of snap bean, lima beall. and cowpeat. Plant Diseatse 69:25-27

W'cinhold, A.R. \& Sinclair, J.B. (1996). Rhizoctonial solani: penctration, colonization. and host response. In: Sneh. B.: Jabaji-Hare, S.; Neate, S. \& Dijst. G. eds. Rhizoctonia species: taxonomy, molecular biology. ecology, pathology and disease control. Kluwer Academic Publishers, Dordrecht. pp. 16.3-174

Williams, R.J. (1975). Control of cowpea seedling mortality in southern Nigeria. Plant Diseatse Reporter 59: 245-248

Zambolim, I.; ('ost:a, II. \& Viale, F.X.R. (1997). Frijol comum(I'haseolus vulgaris L.) - Controle de doenças: podridão, tombamento e murcha catusades por lingos do solo. In: Valc, F....R. \& Zambolim, L. eds. Controle de doençals de plantas: grandes culturas. v.1. Iniversidade Federal de Vï̧osa Ministério da Agricultura e do Abaststecimento. Viçosa/Brasilia. pp).375-4()1 